\title{
Role of CYP2E1 in Ethanol-Induced Oxidant Stress, Fatty Liver and Hepatotoxicity
}

\author{
Arthur l. Cederbaum \\ Department of Pharmacology and Systems Therapeutics, Mount Sinai School of Medicine, New York, N.Y., USA
}

\author{
Key Words \\ CYP2E1 • Chronic ethanol administration • Fatty liver • \\ Oxidative stress $\cdot$ Hepatotoxicity
}

\begin{abstract}
Background/Aims: Several pathways contribute to mechanisms by which ethanol induces oxidant stress. While some studies support a role for cytochrome P450 2E1 (CYP2E1), others do not. There is a need to develop oral models of significant ethanol-induced liver injury and to evaluate the possible role of CYP2E1 in ethanol actions in such models. Methods: We evaluated chronic ethanol-induced liver injury, steatosis and oxidant stress in wild-type (WT) mice, CYP2E1 knockout (KO) mice and in humanized CYP2E1 knockin (KI) mice, where the human $2 \mathrm{E} 1$ was added back to mice deficient in the mouse 2E1. WT mice and CYP2E1 KO and KI mice (both provided by Dr. F. Gonzalez, NCl) were fed a high-fat Lieber-DeCarli liquid diet for 3 weeks; pair-fed controls received dextrose. Results: Ethanol produced fatty liver and oxidant stress in WT mice, but liver injury (transaminases, histopathology) was minimal. Ethanol-induced steatosis and oxidant stress were blunted in the KO mice (no liver injury) but restored in the KI mice. Significant liver injury was produced in the ethanol-fed $\mathrm{KI}$ mice with elevated transaminases and necrosis. This liver injury in the KI mice was associated with elevated oxidant stress and elevated levels of the human CYP2E1 compared to levels of the mouse 2E1 in WT mice. Activation of JNK was observed in the ethanol-fed KI mice compared to the other groups. Fatty liver in WT and KI mice was associated with lower levels of lipolytic PPAR- $\alpha$. No
\end{abstract}

\section{KARGER}

(๑) 2011 S. Karger AG, Basel

Fax +4161306 1234

E-Mail karger@karger.ch

www.karger.com
Accessible online at: www.karger.com/ddi such changes were found in the ethanol-fed KO mice. Conclusions: These results show that CYP2E1 plays a major role in ethanol-induced fatty liver and oxidant stress. Restoring CYP2E1 in the CYP2E1 KO mice restores ethanol-induced fatty liver and oxidant stress.

Copyright $\odot 2011$ S. Karger AG, Basel

\section{Introduction}

The ability of acute and chronic ethanol treatment to increase production of reactive oxygen species and enhance peroxidation of lipids, proteins, and DNA has been demonstrated in a variety of systems, cells, and species, including humans. The mechanism(s) by which alcohol causes cell injury is (are) still not clear. A major mechanism that is a focus of considerable research is the role of lipid peroxidation and oxidative stress in alcohol toxicity. Many pathways have been suggested to play a key role on how ethanol induces 'oxidative stress' [1-4]. Our laboratory has focused on ethanol induction of CYP2E1 [3,5-7]. It is likely that several, indeed many, systems contribute to the ability of ethanol to induce a state of oxidative stress.

CYP2E1 is of interest because of its ability to metabolize and activate many toxicologically important substrates including ethanol, carbon tetrachloride, acetaminophen, and $\mathrm{N}$-nitrosodimethylamine to more toxic products [8-13]. Major interest in CYP2E1 reflects the ability of this enzyme to oxidize ethanol, to generate reactive products from ethanol oxidation, e.g. acetaldehyde 
and the 1-hydroxyethyl radical, to activate various agents $\left(\mathrm{CCl}_{4}\right.$, acetaminophen, benzene, halothane, halogenated alkanes, and alcohols) to reactive products, to generate reactive oxygen radical species and to be induced by ethanol. In the intragastric infusion model of ethanol administration, the ethanol-induced liver pathology has been shown to correlate with CYP2E1 levels and lipid peroxidation [14-18]. Inhibitors of CYP2E1 prevented the elevation of lipid peroxidation and the ethanol-induced liver pathology $[19,20]$. How CYP2E1 may be mediating liver injury beyond oxidative stress is still not clear from these studies.

Understanding the biochemical and toxicological properties of CYP2E1 is important for many reasons, even besides its role in contributing to alcohol-induced liver injury since CYP2E1 is induced under a variety of pathophysiological conditions such as fasting, diabetes, obesity and high-fat diet [21-24] and by drugs [8, 10-13]. Non-alcohol-induced steatohepatitis (NASH) causes steatosis, liver cell injury, inflammation and variable necrosis. NASH is associated with obesity, type 2 diabetes and hyperlipidemia, conditions in which CYP2E1 is induced $[25,26]$.

Thurman and colleagues [27-29] have suggested that CYP2E1 may not play a role in alcohol liver injury based on studies with gadolinium chloride or CYP2E1 knockout (KO) mice or a broad-spectrum P450 inhibitor aminobenzotriazole. Induction by ethanol of other CYPs or of the reductase in the CYP2E1 KO mice might have served as alternative sources of oxidative stress, especially in the absence of CYP2E1 [30]. Clearly, further studies are necessary to resolve the above discrepancies. As mentioned earlier, it is likely that several mechanisms contribute to alcohol-induced liver injury, and that ethanol-induced oxidant stress is likely to arise from several sources, including CYP2E1, mitochondria and activated Kupffer cells. Bradford et al. [31] reported that CYP2E1, but not NADPH oxidase, is required for ethanol-induced oxidative DNA damage in rodent liver, and concluded that CYP2E1 is required for the induction of oxidative stress to DNA and may play a key role in ethanol-associated hepatocarcinogenesis.

Almost all the above studies assessing the role of CYP2E1 in alcoholic liver disease involve the intragastric infusion model where significant liver injury occurs [14-17, 27-29, 31]. Although much valuable information has been gained from this model, it is complex, requires special expertise, is stressful, and is not accessible to most researchers. There is a need to develop oral models of ethanol treatment which result in the production of signifi- cant liver injury. Rodents administered Lieber-DeCarli alcohol-containing liquid diets [32] developed steatosis and oxidative stress in the liver, but significant liver injury beyond steatosis generally does not occur. A modified low-carbohydrate ethanol liquid diet caused mild liver injury in rats [33-36]. Nanji's group [37] recently reported the development of alcoholic liver disease in a voluntary feeding regimen consisting of fish oil and ethanol in female rats. In all these oral models, ALT levels were elevated about 2 -fold by the chronic ethanol feeding. Although CYP2E1 was elevated by ethanol in all these models, whether CYP2E1 played a role in the elevated ALT and oxidative stress or the steatosis was not directly evaluated.

Fatty liver is a uniform and early response of the liver to ethanol consumption. Previously, fatty liver was considered benign, however, it is now known that fatty liver can increase sensitivity to hepatotoxins such as lipopolysaccharide (LPS) and high levels of fatty acids promote lipotoxicity [38-40]. Hence, there is a need to understand the mechanisms responsible for fatty liver production by ethanol. Early hypotheses for mechanisms responsible for fatty liver production by ethanol included redox state changes (elevated NADH) when ethanol was metabolized by alcohol dehydrogenase, elevated formation of acetylCoA from ethanol/acetaldehyde oxidation and impairment of $\beta$-oxidation of fatty acids [41]. Recent studies by Crabb, You and colleagues [42-45] have identified new mechanisms which regulate the synthesis and the oxidation of fatty acids as being central to how ethanol produces fatty liver. Ethanol has been shown to elevate levels of SREBP-1c, a master transcriptional regulator of lipogenic enzymes, but to lower levels of PPAR- $\alpha$, a master transcriptional regulator of lipolytic enzymes. Decreased activation of AMPK by ethanol plays a role in these effects. Is there a role for CYP2E1 in ethanol-induced fatty liver? In the IG infusion model, Kono et al. [28] reported that after feeding ethanol for 4 weeks, there was no difference in ethanol-induced steatosis between CYP2E1 KO mice and wild-type (WT) mice. Similarly, in this model, Wan et al. [46] reported that ethanol infusion for 21 days promoted fat accumulation in CYP2E1 KO mice but not in the WT mice. No explanation or mechanism was provided for these unusual observations. In contrast, the CYP2E1 inhibitor chlormethiazole (CMZ) was reported to blunt ethanol-induced fatty liver in the IG model [20]. We are not aware of any studies providing direct evidence for or against a role for CYP2E1 in fatty liver produced in oral models of ethanol consumption. Studies described below were designed to further evaluate a pos- 
sible role of CYP2E1 in ethanol-induced fatty liver and to assess downstream factors which may play a role in the molecular mechanisms by which CYP2E1 promotes development of fatty liver by ethanol.

\section{Methods}

Animals and Ethanol Treatment

SV129-background CYP2E1 KO [47] and humanized CYP2E1 transgenic (KI) mice $[48,49]$ were kindly provided by Dr. Frank J. Gonzalez (Laboratory of Metabolism, National Cancer Institute, Bethesda, Md., USA), and breeding colonies established at Mount Sinai. Female SV129 WT mice were purchased from Charles River Laboratory. All mice were housed in temperaturecontrolled animal facilities with 12-hour light/12-hour dark cycles and were permitted consumption of tap water and Purina standard chow ad libitum until being fed the liquid diets. The mice received humane care, and experiments were carried out according to the criteria outlined in the Guide for the Care and Use of Laboratory Animals and with approval of the Mount Sinai Animal Care and Use Committee.

Female WT, KO, and KI mice were initially fed the control liquid dextrose for 3 days to acclimate them to the liquid diet. Afterward, the mice were fed either the liquid ethanol diet or the control liquid dextrose diet, as described by Lieber and DeCarli [50] for 3 weeks. The content of ethanol was gradually increased every 3-4 days from $10 \%(1.77 \%[\mathrm{v} / \mathrm{v}])$ of total calories to $20 \%(3.54 \%$ [v/v]), $25 \%(4.42 \%$ [v/v]), 30\% (5.31\% [v/v]), and finally $35 \%$ of total calories $(6.2 \%[\mathrm{v} / \mathrm{v}])$. The control mice were pair-fed the control dextrose diet on an isoenergetic basis. The ethanol-fed mice had access to their rations ad libitum, and the conditions of WT, KO, and humanized transgenic mice were comparable. The amount of food consumed by CYP2E1 KO mice, KI mice and WT mice was approximately the same.

No mice died in any group after 3 weeks of feeding with the control or ethanol-containing diet. Before being sacrificed, the mice were fasted overnight and body weight was measured. Blood was collected and serum was separated. Serum ethanol levels were similar between the three groups of ethanol-fed mice at sacrifice. Whole liver was removed and liver weight measured; the liver was rapidly excised into fragments and washed with cold saline, and one aliquot of tissue was placed in $10 \%$ formalin solution for paraffin blocking. Another piece was placed in Tissue-Tek OCT compound, and was frozen and cut into $10-\mu \mathrm{m}$ frozen sections for Oil Red $\mathrm{O}$ staining and for immunohistochemistry assays. The remaining aliquots were stored at $-80^{\circ} \mathrm{C}$ for further assays.

\section{Assays}

Liver sections were stained with HE for pathological evaluation. Steatosis was quantified as the percentage of cells containing fatty droplets. Necroinflammation was quantified as the number of clusters of $\geq 5$ inflammatory cells $/ \mathrm{mm}^{2}$. Five $200 \times$ fields per liver were examined (one $200 \times$ field area $=0.95 \mathrm{~mm}^{2}$ ). Serum ALT, AST, and ethanol levels, hepatic GSH, thiobarbituric acid reactive substances (TBARS), triglyceride and free fatty acid levels, immunohistochemistry, immunoblots, microsomal oxidation of $p$-nitrophenol (PNP) and other biochemical assays were carried out as previously described $[51,52]$.

\section{Results}

SV129 WT mice, CYP2E1 KO mice and humanized CYP2E1 KI mice were fed the high-fat Lieber-DeCarli ethanol liquid diet over a 3-week period. Mice were pairfed with dextrose controls. There was no change in body weight in any group over the 3 -week feeding period. Because of concerns of extrapolating results with mouse enzymes to human enzymes and to overcome species differences in P450 expression, and specificity between rodents and humans, transgenic humanized mouse models expressing major P450 enzymes were developed by Cheung and Gonzalez [49]. The human P450 transgene was introduced onto the corresponding null mouse background [47] to create a humanized P450 mouse in the absence of the corresponding mouse P450 [48]. Therefore, functional activities and differences between human and mouse CYPs can be directly compared with this model. In the humanized CYP2E1 KI mouse [48], some differences were observed compared to the WT mice expressing mouse CYP2E1 such as rates of PNP (but not chlorzoxazone) oxidation and sensitivity to toxicity by acetaminophen. A major goal of the current report was to validate that any changes in fatty liver or oxidant stress produced by chronic ethanol feeding in CYP2E1 KO mice was indeed due to the deficiency in CYP2E1 by evaluating whether such actions by ethanol are restored when CYP2E1 is restored in the KO mice. Another important goal was to study whether human CYP2E1 could effectively mimic mouse CYP2E1 in contributing to ethanolinduced fatty liver, oxidant stress and liver injury and thus allow assessment of the human CYP2E1 to modulate actions of chronic ethanol administration in a whole animal model system.

\section{Levels of CYP2E1}

Oxidation of PNP to $p$-nitrocatechol, a representative reflection of CYP2E1 catalytic activity, was increased about 2-fold in the ethanol-fed WT mice compared to dextrose-fed mice (fig. 1). As expected, rates of PNP oxidation were low in the CYP2E1 KO mice (the low rates are probably a reflection of PNP oxidation by other CYPs, e.g. CYP3A). Rates of PNP oxidation were highest in the ethanol-fed CYP2E1 KI mice, being about 7-fold higher than the dextrose-fed CYP2E1 KI mice and about 3-fold higher than the ethanol-fed WT mice (fig. 1). Immunoblot analysis showed the expected low levels of CYP2E1 in the $\mathrm{KO}$ mice, an approximate 2 -fold increase in the level of CYP2E1 (CYP2E1/ $\beta$-actin ratio) in ethanol-fed WT compared to dextrose-fed WT mice, and highest 


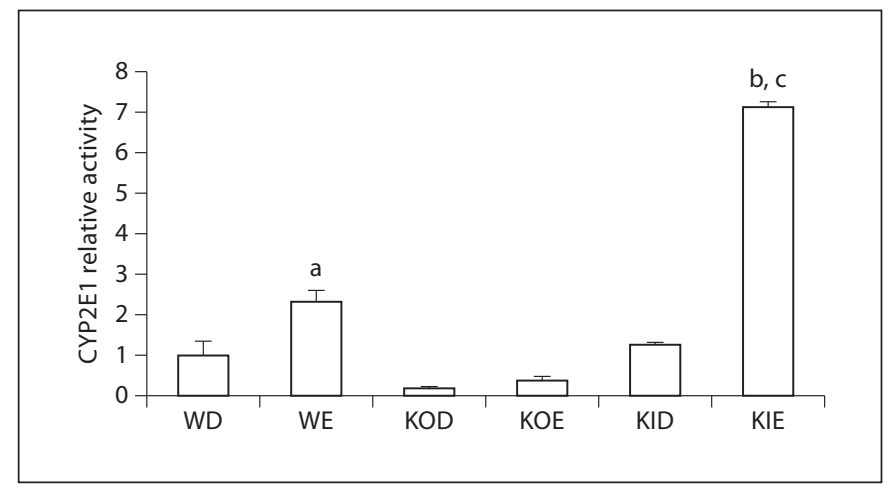

Fig. 1. Catalytic activity of CYP2E1. Activity was assayed from the oxidation of PNP by microsomes isolated from WT dextrose-fed (WD) and ethanol-fed (WE) mice, or from KO dextrose-fed (KOD) and $\mathrm{KO}$ ethanol-fed (KOE) mice or from KI dextrose-fed (KID) and KI ethanol-fed (KIE) mice. Results are from 3 to 6 pairs of mice in each group. ${ }^{\mathrm{a}} \mathrm{p}<0.01$ compared to WD; ${ }^{\mathrm{b}} \mathrm{p}<0.01 \mathrm{com}-$ pared to KID; ${ }^{\mathrm{c}} \mathrm{p}<0.01$ compared to WE.

levels of CYP2E1 in the ethanol-fed CYP2E1 KI mice (CYP2E1/ $\beta$-actin ratio of 6 ). Immunohistochemistry paralleled the results on PNP oxidation and the immunoblots (not shown). The chronic ethanol feeding elevated CYP2E1 in WT mice, and more strikingly in KI mice, in the pericentral zone of the liver acinus. No CYP2E1 was detectable in the ethanol- or dextrose-fed $\mathrm{KO}$ mice.

\section{Ethanol-Induced Fatty Liver}

HE staining showed that ethanol produced fatty liver in the WT mice after the 3-week feeding period. This ethanol-induced steatosis was blunted in the CYP2E1 KO mice but at least partially restored in the CYP2E1 KI mice. Oil Red O staining showed that prominent lipid droplets were observed in frozen liver sections from WT mice fed ethanol whereas only a few lipid droplets were present in the ethanol-fed CYP2E1 KO mice. Oil Red O staining was restored in the CYP2E1 KI mice (data not shown). Triglyceride levels were elevated in livers from all ethanol-fed mice compared to dextrose-fed mice (fig. 2). Triglyceride levels were highest in the ethanol-fed WT mice, followed by the ethanol-fed CYP2E1 KI mice, and lowest in the ethanol-fed CYP2E1 KO mice (fig. 2). Levels of free fatty acids were also highest in livers from the ethanol-fed WT mice, lowest in the ethanol-fed CYP2E1 KO mice and intermediate in the ethanol-fed CYP2E1 KI mice. The ethanol feeding modestly elevated levels of free fatty acids in the WT and CYP2E1 KI mice but not in the CYP2E1 KO mice. The steatosis score confirmed that ethanol produced fatty liver in the WT and the KI mice

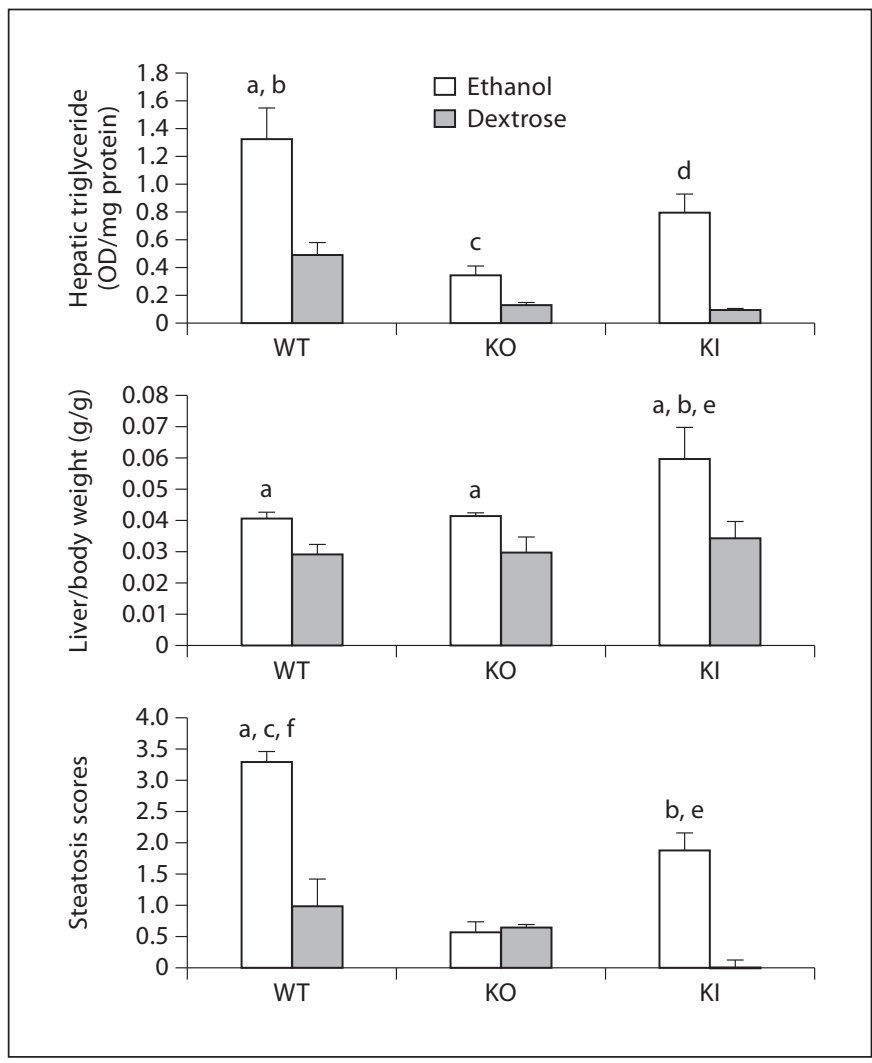

Fig. 2. Ethanol-induced steatosis. Hepatic triglycerides were assayed in livers from dextrose-fed and ethanol-fed WT, KO and KI mice. The steatosis score was derived in a blind fashion from the histopathology. ${ }^{a} \mathrm{p}<0.01$ compared to dextrose-fed; ${ }^{\mathrm{b}} \mathrm{p}<0.01$ compared to ethanol-fed $\mathrm{KO} ;{ }^{\mathrm{c}} \mathrm{p}<0.05$ compared to dextrose-fed $\mathrm{KO} ;{ }^{\mathrm{d}} \mathrm{p}<0.05$ compared to ethanol-fed WT; ${ }^{\mathrm{e}} \mathrm{p}<0.05$ compared to ethanol-fed KO; ${ }^{\mathrm{f}} \mathrm{p}<0.05$ compared to ethanol-fed KI.

but not the $\mathrm{KO}$ mice (fig. 2). These results are supportive of a role of CYP2E1 in ethanol-induced steatosis.

\section{Role of CYP2E1 in Ethanol-Induced Hepatotoxicity}

Serum ALT and AST levels were increased $50-100 \%$ in the ethanol-fed WT and CYP2E1 KO mice but were elevated about 4 -fold in the ethanol-fed CYP2E1 KI mice (fig. 3). HE staining (not shown) showed lipid droplets in the ethanol-fed WT mice compared to the dextrose-fed mice but no cellular necrosis or degeneration. No lipid droplets were found in the ethanol-fed CYP2E1 KO mice and pathology was normal. Steatosis reappeared in the ethanol-fed CYP2E1 KI mice; importantly, areas with cell swelling, focus necrosis and inflammatory infiltration were observed in the ethanol-fed KI mice. Necroinflammatory scores were elevated only for the ethanol-fed KI mice (fig. 3). We did not observe any increase in the activ- 


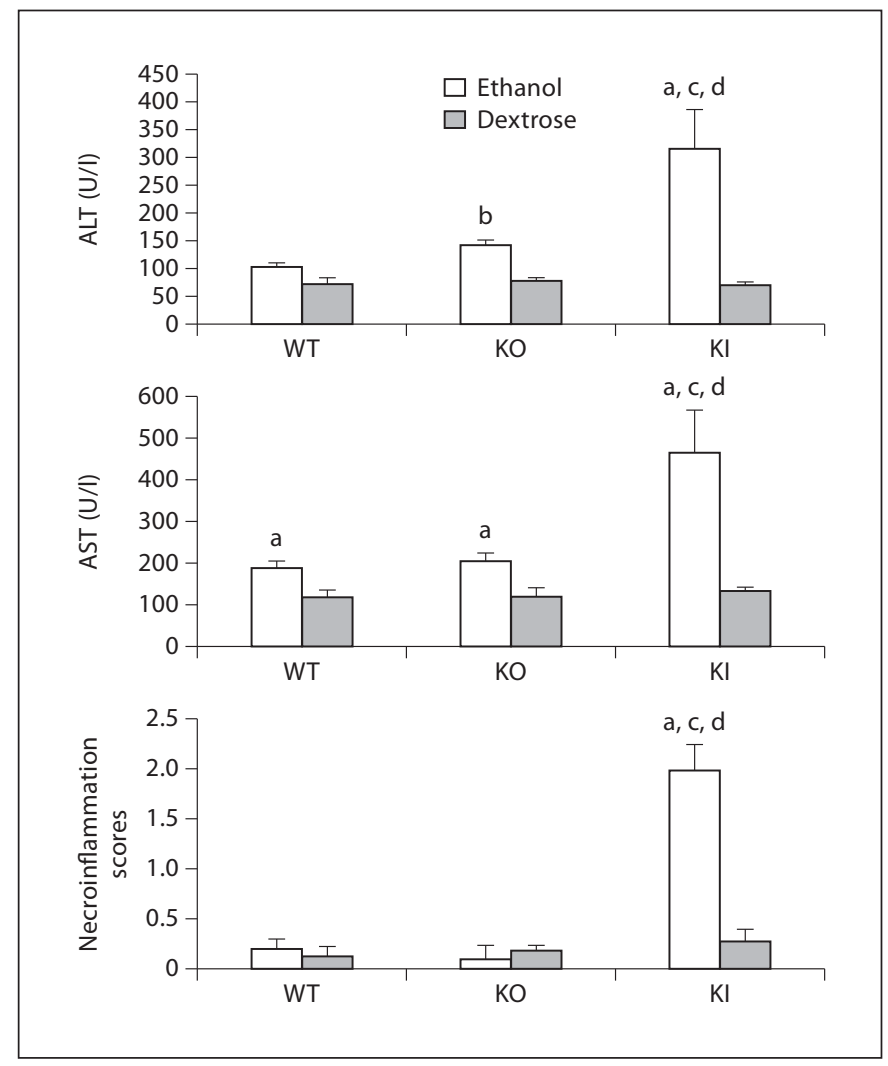

Fig. 3. Ethanol-induced hepatotoxicity. ALT and AST were assayed in the serum derived from dextrose- and ethanol-fed WT, KO and KI mice. ${ }^{\mathrm{a}} \mathrm{p}<0.05 ;{ }^{\mathrm{b}} \mathrm{p}<0.01$ compared to dextrose-fed mice; ${ }^{\mathrm{c}} \mathrm{p}<0.05$ compared to WT ethanol-fed mice; ${ }^{\mathrm{d}} \mathrm{p}<0.05$ compared to $\mathrm{KO}$ ethanol-fed mice.

ity of caspase 3 or caspase 9 in the ethanol-fed CYP2E1 KI mice compared to dextrose control nor was there a significant increase in TUNEL staining suggesting necrotic but not apoptotic liver injury was occurring in the ethanol-fed humanized CYP2E1 KI mice. Interestingly, while hepatotoxicity was highest in the CYP2E1 KI mice, fatty liver was highest in WT mice followed by the CYP2E1 KI mice.

\section{Ethanol-Induced Oxidant Stress}

CYP2E1 plays a central role in how ethanol produces oxidant stress in the liver. Chronic ethanol consumption by WT mice elevated hepatic TBARS levels (a reflection of lipid peroxidation) about 2-fold, whereas hepatic GSH levels were decreased about 50\% (fig. 4). TBARS were not elevated nor was GSH depleted in the ethanol-fed CYP2E1 KO mice (fig. 4). The increase in lipid peroxidation was 'restored' in the ethanol-fed CYP2E1 KI mice
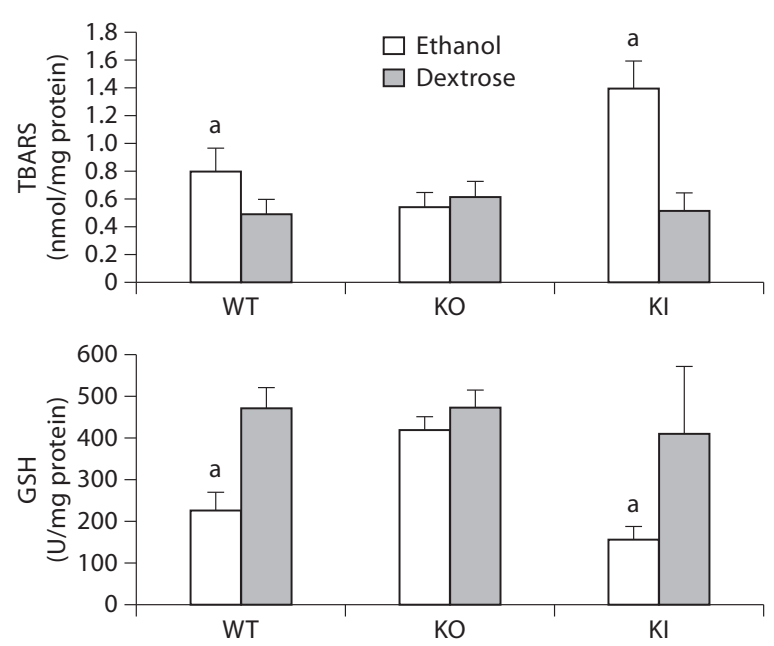

Fig. 4. Ethanol-induced oxidative stress. Levels of TBARS and glutathione (GSH) were assayed in liver lysates from dextroseand ethanol-fed WT, KO and KI mice. Results are from 3 to 6 pairs of mice in each group. ${ }^{a} \mathrm{p}<0.05$ compared to dextrose-fed mice.

(2.8-fold increase in TBARS) as was the depletion of GSH (65\% decrease) (fig. 4). The elevation of TBARS and the decline in GSH were greater in the CYP2E1 KI mice than the WT mice, which may reflect the elevated level of CYP2E1 in the ethanol-fed KI mice. Formation of 3-nitrotyrosine protein adducts is one assay for detection of peroxynitrite formation [28]. Low levels of 3-nitrotyrosine (3-NT) staining were observed in livers from the ethanol-fed WT and KO mice, but a more intense staining was observed in livers from the ethanol-fed KI mice. 4-Hydroxynonenal (4-HNE) protein adducts as detected by immunohistochemistry were elevated in WT mice and to a greater extent in KI mice as compared to dextrose controls. Thus, levels of oxidant stress induced by ethanol appeared to follow a similar pattern of induction of CYP2E1 by ethanol, being highest in the CYP2E1 KI mice, intermediate in the WT mice, and lowest in the CYP2E1 KO mice. We believe that exacerbation of ethanol-induced oxidant stress plays a key role in the hepatotoxicity and contributes, at least in part, to the steatosis found in the ethanol-fed CYP2E1 KI mice.

\section{Ethanol Activates JNK in WT and CYP2E1 KI Mice} but Not CYP2E1 KO Mice

Activation of JNK has been shown to be important in many models of hepatotoxicity [53-57] including 

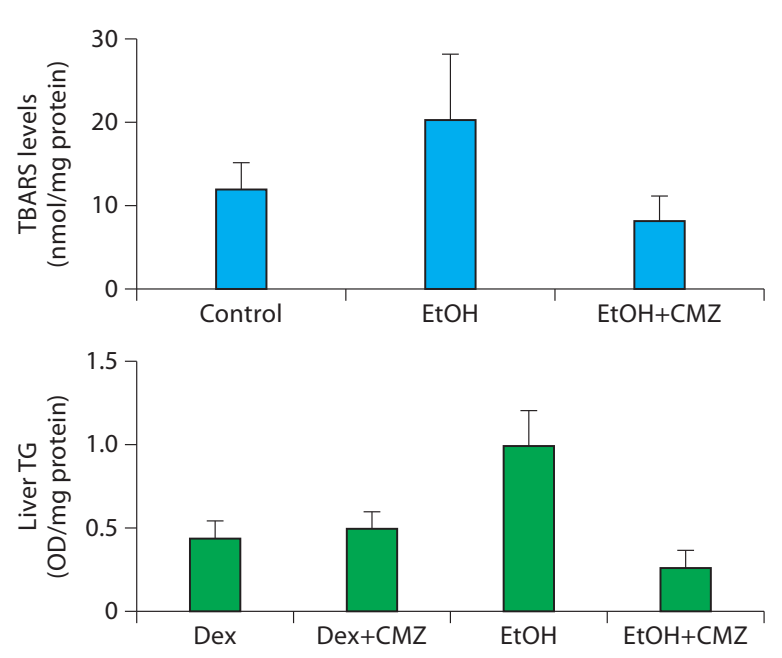

Triglyceride (TG) levels in WT mice livers after treatment with CMZ
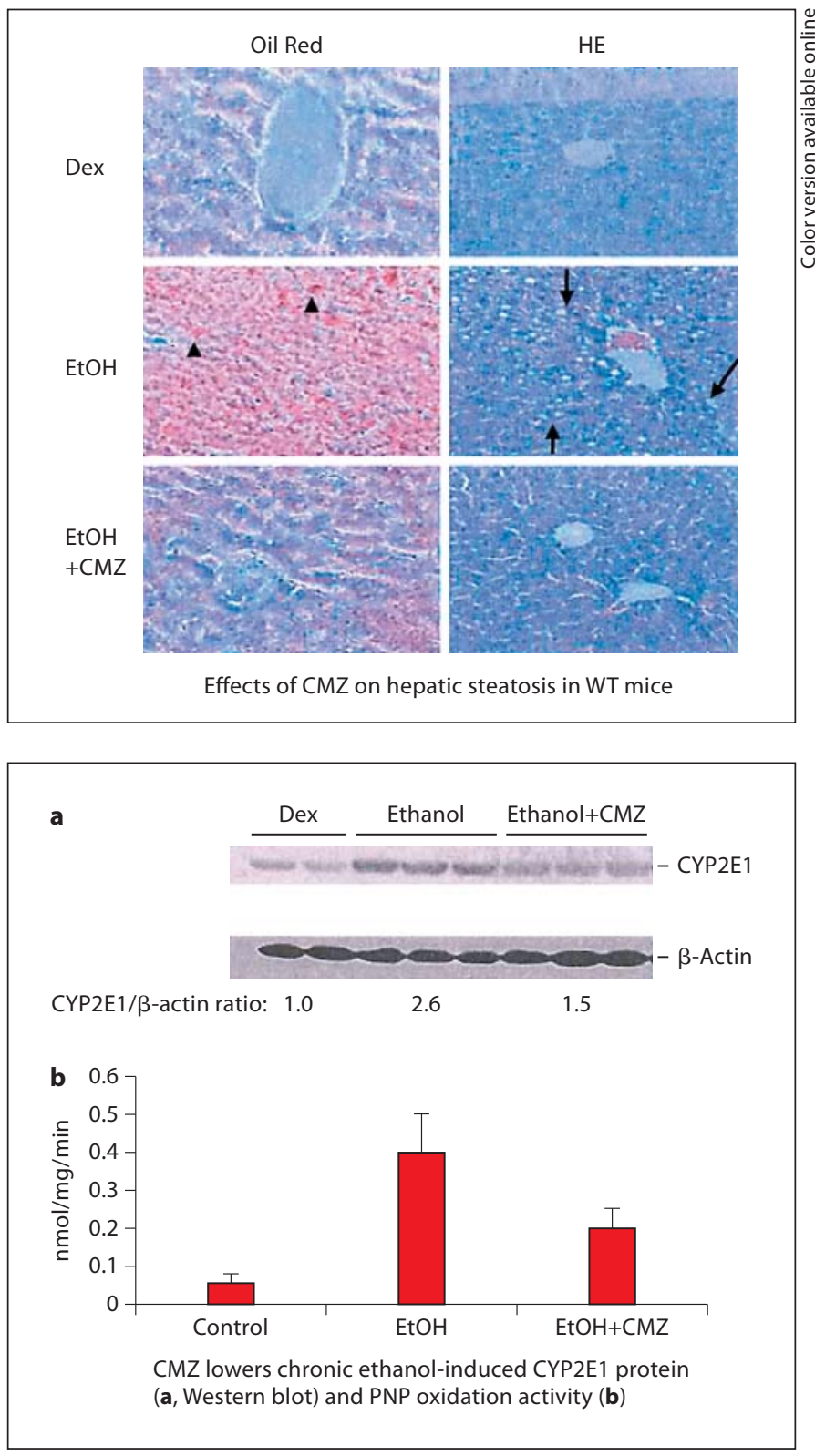

While preliminary, the significance of this activation of JNK2 or of JNK 1 in ethanol hepatotoxicity or hepatic fatty liver, if any, will be an important goal of future studies. We did not observe any activation of p38 MAP kinase by ethanol in any group.

\section{Effect of CMZ on Ethanol-Induced Fatty Liver, CYP2E1 and Oxidant Stress}

The CYP2E1 inhibitor CMZ was used to evaluate the role of CYP2E1 in ethanol-induced fatty liver and oxidant stress. SV129 WT mice were fed the dextrose or ethanol 
Lieber-DeCarli diet for 2 weeks. CMZ (Sigma, $50 \mathrm{mg} / \mathrm{kg}$ i.p.) was injected every other day over the 2 -week experiment; controls were injected with saline. Moderate steatosis was found after 2 weeks of ethanol feeding as detected by HE or Oil Red O staining (fig. 5). Liver triglycerides were elevated about 2 -fold (fig. 5). CMZ treatment lowered the ethanol-induced steatosis and decreased triglyceride levels. CMZ treatment lowered the elevated levels of CYP2E1 and the increased CYP2E1 catalyzed oxidation of PNP produced by the ethanol feeding (fig. 5). Feeding ethanol to WT mice for 2 weeks increased hepatic TBARS levels 2 -fold and this increase was completely prevented by CMZ (fig. 5). To further substantiate the effect of CMZ on ethanol-induced oxidative/nitrosative stress, immunohistochemical analysis of 3-NT and 4 -HNE protein adduct formation was evaluated. The ethanol feeding produced an increase in formation of 3-NT and 4-HNE protein adducts and CMZ treatment inhibited these increases (data not shown). These results show the effectiveness of the CYP2E1 inhibitor CMZ in blunting ethanol-induced oxidative stress and ethanol-induced fatty liver.

\section{Discussion}

As expected, ethanol produced fatty liver in WT mice using the high-fat Lieber-DeCarli model. This fatty liver was blunted in CYP2E1 KO mice as compared to WT mice as evident by histopathology, Oil Red O staining and biochemical assay of hepatic triglyceride levels in livers from WT mice fed ethanol compared to CYP2E1 KO mice fed ethanol. We believe two important new points arise from these results. First, it is the absence of CYP2E1 in the $\mathrm{KO}$ mice and not secondary effects associated with the KO that plays a major role in the blunting of the ethanol-induced fatty liver in the $\mathrm{KO}$ mice since restoring the presence of CYP2E1 in these mice restores the fatty liver. Second, human CYP2E1 was the CYP2E1 which restores the ethanol-induced steatosis, which indicates that human CYP2E1 can potentiate development of fatty liver in an oral model of chronic ethanol administration. This suggests that results on fatty liver extrapolated from rodent models of ethanol intake can be extrapolated to human models of ethanol intake with respect to effects of CYP2E1.

Associated with the fatty liver induced by ethanol was an increase in oxidative/nitrosative stress as reflected by the increase in TBARS, 3-NT and 4-HNE staining, and the decrease in GSH levels in the WT mice fed ethanol.
CYP2E1 is suggested to be a major contributor to ethanol-induced oxidant stress. Ethanol caused oxidative stress in WT mice, but the ethanol-induced oxidative stress was decreased in CYP2E1 KO mice. It is the absence of CYP2E1 in the CYP2E1 KO mice that plays a major role in blunting of the ethanol-induced oxidant stress since restoring CYP2E1 restores oxidative/nitrosative stress. As discussed above for fatty liver, human CYP2E1 was the CYP2E1 which restores the oxidative/ nitrosative stress indicating that human CYP2E1 can potentiate development of oxidative/nitrosative stress in an oral model of chronic ethanol administration.

It appears that ethanol-induced steatosis may be related to oxidative stress as shown by Diluzio [62] who reported that antioxidants can prevent the ethanol-induced fatty liver, and studies showing that overexpression of the manganese superoxide dismutase- 2 can partially reduce the ethanol steatosis [63]. CYP2E1-generated ROS contributes to the ethanol-induced oxidant stress which may explain its role in the ethanol-induced steatosis. However, mechanisms for ethanol-induced steatosis are likely to be more complex than increases in CYP2E1/cytokines/oxidant stress, e.g. ethanol did not induce fatty liver in C1q KO mice despite elevating CYP2E1, TNF- $\alpha$, IL- 6 and oxidant stress, and the authors suggested a role for the classical complement pathway in contributing to the pathogenesis of ethanol-induced liver injury [64]. Blockade of PPAR- $\alpha$ function by chronic ethanol administration plays a key role in the mechanism of ethanol-induced steatosis [65] as PPAR- $\alpha \mathrm{KO}$ mice fed ethanol develop extensive fatty liver and liver injury [66], whereas PPAR- $\alpha$ activating ligands such as clofibrate [67] or WY14643 [65] decrease the ethanol fatty liver. We found that levels of PPAR- $\alpha$, and a downstream target of PPAR- $\alpha$, acyl-CoA oxidase, were decreased more than $50 \%$ by ethanol administration to WT mice and CYP2E1 KI mice, but no effect was found with the CYP2E1 KO mice. This suggests the ethanol-induced decline in PPAR- $\alpha$ may be due, at least in part, to CYP2E1, or more likely, CYP2E1-derived ROS.

Significant liver injury generally does not occur in most oral models of ethanol intake, and there is a need to develop such models for mechanistic and preventive/ therapeutic designs. ALT, AST and necroinflammatory scores were higher in ethanol-fed CYP2E1 KI mice compared to WT and CYP2E1 KO mice. CYP2E1 levels and activity were about 3-fold higher in the CYP2E1 KI mice compared to the WT mice after the ethanol feeding, suggesting the elevated liver injury may be due to the elevated content of CYP2E1, although the possibility that the 
human CYP2E1 is more reactive than the mouse CYP2E1 in promoting this injury remains to be evaluated. The elevated liver injury is associated with an elevated oxidative/nitrosative stress in the ethanol-fed CYP2E1 KI mice and we speculate that the latter plays a key role in the former. This will be evaluated in future studies examining the effects of antioxidants such as $\mathrm{N}$-acetylcysteine, vitamins $\mathrm{E}$ and $\mathrm{C}$, and inhibitors of the inducible nitric oxide synthase, e.g. $1400 \mathrm{~W}$. The elevated liver injury is also associated with an increase in activation of the JNK mitogen-activated protein kinase. Activation of JNK has been shown to be important in many models of hepatotoxicity [53-57], including CYP2E1 potentiation of LPS or TNF- $\alpha$ liver injury [58-61]. Future experiments will evaluate the ability of an inhibitor of JNK activation such as SP600125 to prevent the chronic ethanol-induced liver injury in the CYP2E1 KI mice. SP600125 was effective in preventing the CYP2E1 potentiation of LPS or TNF- $\alpha$ liver injury [59]. We speculate the elevated oxidative/nitrosative stress in ethanol-fed KI mice may play a role in the activation of JNK, e.g. the upstream MAPKKK ASK-1 is activated when its inhibitor thioredoxin-1 is oxidized by ROS, and dissociates off ASK-1, which then allows ASK-1 to activate downstream MAPKKs and subsequently JNK
$[68,69]$. ROS also inactivate MAPK phosphatases which dephosphorylate activated MAPK such as JNK [70]. Although other models of hepatotoxicity produced by overexpression of CYP2E1 have been reported, e.g. a transgenic model of CYP2E1 overexpression [71, 72], or administration of an adenovirus expressing CYP2E1 to mice [73], the advantage of the CYP2E1 KI model is that the actions of the human CYP2E1 can be studied in the total absence of the mouse CYP2E1, thus allowing assessment of the role of human CYP2E1 in ethanol-induced oxidative stress and liver injury.

\section{Acknowledgements}

Studies in the author's laboratory were carried out by Drs. Defeng Wu, Yongke Lu, Xiaodong Wang, Jing Xiang Bai, Jian Zhuge and Stephen Ward and were supported by USPHS grants RO1 AA-017425 and AA-018790 from the National Institute on Alcohol Abuse and Alcoholism.

\section{Disclosure Statement}

There are no conflicts of interest to disclose.

\section{References}

1 Nordman R, Riviere C, Rouach H: Implication of free radical mechanisms in ethanolinduced cellular injury. Free Radic Biol Med 1992;12:219-240.

$>2$ Bondy SC: Ethanol toxicity and oxidative stress. Toxicol Lett 1992;63:231-242.

-3 Cederbaum AI: Introduction serial review: alcohol, oxidative stress and cell injury. Free Radic Biol Med 2001;31:1524-1526.

-4 Arteel GE: Oxidants and antioxidants in alcohol-induced liver disease. Gastroenterology 2003;124:778-790.

5 Caro AA, Cederbaum AI: Oxidative stress, toxicology and pharmacology of CYP2E1. Annu Rev Pharmacol Toxicol 2004;44:2742.

6 Kessova I, Cederbaum AI: CYP2E1: biochemistry, toxicology and regulation. Curr Mol Med 2003;3:509-518.

7 Jimenez-Lopez JM, Cederbaum AI: CYP2E1-dependent oxidative stress and toxicity: role in ethanol-induced liver injury. Expert Opin Drug Metab Toxicol 2005;1:671-685.

-8 Raucy JL, Kraner JC, Lasker JM: Bioactivation of halogenated hydrocarbons by cytochrome P4502E1. Crit Rev Toxicol 1993;2: $1-20$.
9 Bolt M, Koos PH, Their R: The cytochrome P450 isoenzyme CYP2E1 in the biological processing of industrial chemicals. Int Arch Occup Environ Health 2003;76:174-185.

10 Koop DR: Oxidative and reductive metabolism by cytochrome P4502E1. FASEB J 1992; 6:724-730.

11 Song BJ, Cederbaum AI, Koop DR, Ingelman-Sundberg M, Nanji A: Ethanol-inducible cytochrome P450 (CYP2E1): biochemistry, molecular biology and clinical relevance. Alcohol Clin Exp Res 1996;20(suppl):138A$146 \mathrm{~A}$.

12 Tanaka E, Terada M, Misawa S: Cytochrome P450 2E1: its clinical and toxicological role. J Clin Pharm Ther 2000;25:165-175.

13 Gonzalez FJ: Role of cytochromes P450 in chemical toxicity and oxidative stress: studies with CYP2E1. Mutat Res 2005;569:101110.

14 French SW, Morimoto M, Reitz RC, Koop D, Klopfenstein B, Estes K, Clot P, IngelmanSundberg M, Albano E: Lipid peroxidation, CYP2E1 and arachidonic acid metabolism in alcoholic liver disease in rats. J Nutr 1997; 127:907S-911S.
15 Castillo T, Koop DR, Kamimura S, Triadafilopoulos G, Tsukamoto H: Role of cytochrome P4502E1 in ethanol, carbon tetrachloride and iron-dependent microsomal lipid peroxidation. Hepatology 1992;16:992996.

16 Morimoto M, Zern MA, Hagbjork AL, Ingelman-Sundberg M, French SW: Fish oil, alcohol and liver pathology: role of cytochrome P450 2E1. Proc Soc Exp Biol Med 1994;207: 197-205.

-17 Nanji AA, Zhao S, Sadrzadeh SMH, Dannenberg AJ, Tahan SR, Waxman DJ: Markedly enhanced cytochrome P4502E1 induction and lipid peroxidation is associated with severe liver injury in fish oil-ethanol-fed rats. Alcohol Clin Exp Res 1994;18:12801285.

18 Tsukamoto H, Lu SC: Current concepts in the pathogenesis of alcoholic liver injury. FASEB J 2001;15:1335-1349.

19 Morimoto M, Hagbjork AL, Wan YJ, Fu PC, Clot P, Albano E, Ingelman-Sundberg M, French SW: Modulation of experimental alcohol-induced liver disease by cytochrome P450 2E1 inhibitors. Hepatology 1995;21: 1610-1617. 
$>20$ Gouillon Z, Lucas D, Li J, Hagbjork AL, French BA, Fu P, Fang C, Ingelman-Sundberg M, Donohue TM Jr, French SW: Inhibition of ethanol-induced liver disease in the intragastric feeding rat model by chlormethiazole. Proc Soc Exp Biol Med 2000; 224:302-308.

-21 Raucy JL, Lasker JM, Kramer JC, Salazer DE, Lieber CS, Corcoran GB: Induction of P450IIE1 in the obese rat. Mol Pharmacol 1991;39: 275-280.

-22 Yun Y, Casazza JP, Sohn DH, Veech RL, Song BJ: Pretranslational activation of cytochrome P450IIE during ketosis induced by a high fat diet. Mol Pharmacol 1992;41:474479.

23 Woodcroft KJ, Hafner MS, Novak RF: Insulin signaling in the transcriptional and posttranscriptional regulation of CYP2E1 expression. Hepatology 2002;35:263-273.

-24 Hong JY, Pan J, Gonzalez FJ, Gelboin HV, Yang CS: The induction of a specific form of cytochrome P450 (P450j) by fasting. Biochem Biophys Res Commun 1987;142:10771083.

25 Weltman MD, Farrell GC, Liddle C: Increased hepatocytes CYP2E1 expression in a rat nutritional model of hepatic steatosis and inflammation. Gastroenterology 1996;111: 1645-1653.

-26 Weltman MD, Farrell GC, Hall P, IngelmanSundberg M, Liddle C: Hepatic cytochrome $\mathrm{P} 4502 \mathrm{E} 1$ is increased in patients with nonalcoholic steatohepatitis. Hepatology 1998;27: 128-133.

-27 Koop DR, Klopfenstein B, Iimuro Y, Thurman RG: Gadolinium chloride blocks alcohol-dependent liver toxicity in rats treated chronically with intragastric alcohol despite the induction of CYP2E1. Mol Pharmacol 1997;51:944-950.

- 28 Kono H, Bradford BU, Yin M, Sulik KK, Koop DR, Peters JM, Gonzalez FJ, McDonald T, Dikalova A, Kadiiska MB, Mason RP, Thurman RG: CYP2E1 is not involved in early alcohol-induced liver injury. Am J Physiol 1999;277:G1259-G1267.

-29 Isayama F, Froh M, Bradford BU, McKim SE, Kadiiska MB, Connor HD, Mason RP, Koop DR, Wheeler MD, Arteel GE: The CYP inhibitor 1-aminobenzotriazole does not prevent oxidative stress associated with alcoholinduced liver injury in rats and mice. Free Radic Biol Med 2003;35:1568-1581.

- 30 Tsukamoto H: CYP2E1 and alcoholic liver disease. Hepatology 2000;32:154-156.

- 31 Bradford BU, Kono H, Isayama F, Kosyk O, Wheeler MD, Akiyama TE, Bleye L, Krausz KW, Gonzalez, FJ, Koop DR, Rusyn I: Cytochrome P4502E1 but not NADPH oxidase is required for ethanol-induced oxidative DNA damage in rodent liver. Hepatology 2005;41: 336-344.

32 Lieber CS: Alcohol and the liver: 1994 update. Gastroenterology 1994;106:1085-1095.
Korourian S, Hakkak R, Ronis MJ, Shelnutt SR, Waldron J, Ingelman-Sundberg M, Badger TM: Diet and risk of ethanol-induced hepatotoxicity: carbohydrate-fat relationships in rats. Toxicol Sci 1999;47:110-117.

-34 Lindros KO, Jarvelainen HA: A new oral low-carbohydrate alcohol liquid diet producing liver lesions: a preliminary account. Alcohol Alcohol 1998;33:347-353.

- 35 Tsukada H, Wang PY, Kaneko T, Wang Y, Nakano M, Sato A: Dietary carbohydrate intake plays an important role in preventing alcoholic fatty liver in the rat. J Hepatol 1998; 29:715-724.

36 Li J, French BA, Riley N, Bardag-Gorce F, Fu P, French SW: Oral low-carbohydrate alcohol liquid diet induces experimental steatohepatitis in the rat. Exp Mol Pathol 2001;71: 132-136.

- 37 Tiptoe GL, Liong EC, Casey CA, Donohue TM, Eagon PK, So H, Leung TM, Fogt F, Nanji AA: A voluntary oral ethanol-feeding rat model associated with necroinflammatory liver injury. Alcohol Clin Exp Res 2008; 32:669-682.

38 Yang SQ, Lin HZ, Lane MD, Clemens M, Diehl AM: Obesity increases sensitivity to endotoxin liver injury: implications for the pathogenesis of steatohepatitis. Proc Natl Acad Sci USA 1997;94:2557-2562.

39 Yang S, Lin H, Diehl AM: Fatty liver vulnerability to endotoxin-induced damage despite NF- $\kappa \mathrm{B}$ induction and inhibited caspase-3 activation. Am J Physiol Gastrointest Liver Physiol 2001;281:G382-G392.

40 Koteish A, Yang S, Lin H, Huang X, Diehl AM: Chronic ethanol exposure potentiates lipopolysaccharide liver injury despite inhibiting Jun N-terminal kinase and caspase3 activation. J Biol Chem 2002;277:1303713044.

41 Purohit V, Gao B, Song BJ: Molecular mechanisms of alcoholic fatty liver. Alcohol Clin Exp Res 2009;33:191-205.

42 You M, Crabb DW: Recent advances in alcoholic liver disease II. Minireview: molecular mechanisms of alcoholic fatty liver. Am J Physiol Gastrointest Liver Physiol 2004; 287:G1-G6.

43 You M, Crabb DW: Molecular mechanisms of alcoholic fatty liver: role of sterol regulatory element-binding proteins. Alcohol 2004;34:39-43.

44 Crabb DW, Liangpunsaku S: Alcohol and lipid metabolism. J Gastroenterol Hepatol 2006;21:S56-S60.

45 Sozio M, Crabb DW: Alcohol and lipid metabolism. Am J Physiol Endocrinol Metab 2008;295:E10-E16.

46 Wan YY, Cai Y, Li J, Yuan Q, French B, Gonzalez FJ, French S: Regulation of peroxisome proliferator-activated receptor $\alpha$-mediated pathways in alcohol fed cytochrome P450 2E1-deficient mice. Hepatol Res 2001;19: 117-130.
47 Lee SS, Buter JT, Pineau T, Fernandez-Salguero P, Gonzalez FJ: Role of CYP2E1 in the hepatotoxicity of acetaminophen. J Biol Chem 1996;271:12063-12067.

48 Cheung C, Yu AM, Ward JM, Krausz JW, Akiyama TE, Feigenbaum L, Gonzalez FJ: The CYP2E1-humanized transgenic mouse: role of CYP2E1 in acetaminophen hepatoxicity. Drug Metab Disp 2005;33:449-457.

49 Cheung C, Gonzalez FJ: Humanized mouse lines and their application for prediction of human drug metabolism and toxicological risk assessment. J Pharmacol Exp Ther 2008; 327:288-299.

50 Lieber CS, DeCarli LM: Animal models of chronic ethanol toxicity. Methods Enzymol 1994;233:585-594.

-51 Lu Y, Zhuge J, Wang X, Bai J, Cederbaum AI Cytochrome P4502E1 contributes to ethanol-induced fatty liver in mice. Hepatology 2008;47:1483-1494.

$52 \mathrm{Wu}$ D, Xu CJ, Cederbaum AI: Role of nitric oxide and nuclear factor- $\kappa \mathrm{B}$ in the CYP2E1 potentiation of TNF- $\alpha$ hepatotoxicity in mice. Free Radic Biol Med 2009;46:480-491.

53 Liu H, Lo CR, Czaja MJ: NF-кB inhibition sensitizes hepatocytes to TNF-induced apoptosis through a sustained activation of JNK and c-Jun. Hepatology 2002;35:772778.

54 Czaja MJ: The future of GI and liver research: editorial perspectives. III. JNK/AP-1 regulation of hepatocyte death. Am J Physiol Gastrointest Liver Physiol 2003;284:G875G879.

55 Henderson NC, Pollock K, Frew J, Mackinnon AC, Flavell RA, Davis R, Sethi T, Simpson $\mathrm{KJ}$ : Critical role of c-jun $\left(\mathrm{NH}_{2}\right)$ terminal kinase in paracetamol-induced acute liver failure. Gut 2001;56:982-990.

56 Gunawan BK, Liu ZX, Han D, Hanawa N, Gaarde WA, Kaplowitz N: c-Jun N-terminal kinase plays a major role in murine acetaminophen hepatotoxicity. Gastroenterology 2006;131:165-178.

57 Bae MA, Pie JE, Song BJ: Acetaminophen induces apoptosis of $\mathrm{C} 6$ glioma cells by activating the c-Jun $\mathrm{NH}_{2}$-terminal protein kinaserelated cell death pathway. Mol Pharmacol 2001;60:847-856.

58 Liu H, Jones BE, Bradham C, Czaja MJ: Increased cytochrome P450 2E1 expression sensitizes hepatocytes to c-Jun-mediated cell death from TNF- $\alpha$. Am J Physiol Gastrointest Liver Physiol 2002;282:G257-G266.

59 Wu D, Cederbaum AI: Cytochrome P4502E1 sensitizes to tumor necrosis factor $\alpha$-induced liver injury through activation of mitogen-activated protein kinases in mice. Hepatology 2008;47:1005-1017.

60 Lu Y, Wang X, Cederbaum AI: Lipopolysaccharide-induced liver injury in rats treated with the CYP2E1 inducer pyrazole. Am J Physiol Gastrointest Liver Physiol 2005; 289:G308-G319. 
61 Lu Y, Cederbaum AI: Enhancement by pyrazole of lipopolysaccharide-induced liver injury in mice: role of cytochrome P450 2E1 and 2A5. Hepatology 2006;44:263-274.

62 Diluzio NR: Prevention of the acute ethanolinduced fatty liver by the simultaneous administration of antioxidants. Life Sci 1964;3 113-118.

-63 Wheeler MD, Nakagami M, Bradford BU, Uesugi T, Mason RP, Connor HD, Dikalova A, Kadiiska M, Thurman RG: Overexpression of manganese superoxide dismutase prevents alcohol-induced liver injury in the rat. J Biol Chem 2001;276:36664-36672.

-64 Cohen JI, Roychowdhury S, McMullen MR, Stavitsky AB, Nagy LE: Complement and alcoholic liver disease: role of Clq in the pathogenesis of ethanol-induced liver injury in mice. Gastroenterology 2010;139:664-674.

65 Fisher M, You M, Matsumoto M, Crabb DW: PPAR agonist treatment reverses PPAR- $\alpha$ dysfunction and abnormalities in hepatic lipid metabolism in ethanol-fed mice. J Biol Chem 2003;278:27997-28004.
66 Nakajima T, Kamijo Y, Tanaka N, Sugiyama E, Tanaka E, Kiyosawa K, Fukushima Y, Peters JM, Gonzalez FJ, Aoyama T: Peroxisome proliferator-activated receptor- $\alpha$ protects against alcohol-induced liver damage. Hepatology 2004;40:972-980.

67 Nanji AA, Dannenberg AJ, Jokelainen K, Bass NM: Alcoholic liver injury in the rat is associated with reduced expression of peroxisome proliferator- $\alpha$ regulated genes and is ameliorated by PPAR- $\alpha$ activation. J Pharmacol Exp Ther 2004;310:417-424.

68 Fujino G, Noguchi T, Matsuzawa A, Yamauchi S, Saitoh M, Takeda K, Ichijoh H: Thioredoxin and TRAF family proteins regulate reactive oxygen species-dependent activation of ASK1 through reciprocal modulation of the N-terminal homophilic interaction of ASK1. Mol Cell Biol 2007;27:8152-8163.

69 Liu H, Nishitoh H, Ichijo H, Kyriakis JM: Activation of apoptosis signal-regulating kinase 1 (ASK1) by tumor necrosis factor receptor-associated factor 2 requires prior dissociation of the ASK1 inhibitor thioredoxin. Mol Cell Biol 2000;20:2198-2208.
70 Kamata H, Honda S, Maeda S, Chang L, Hirata $\mathrm{H}$, Karin $\mathrm{M}$ : Reactive oxygen species promote $\mathrm{TNF} \alpha$-induced death and sustained JNK activation by inhibiting MAP kinase phosphatases. Cell 2005;120:649-661.

71 Morgan K, French SW, Morgan TR: Production of a cytochrome P450 2E1 transgenic mouse and initial evaluation of alcoholic liver damage. Hepatology 2002;36:122-134.

72 Butura A, Nilsson K, Morgan K, Morgan TR, French S, Johanssson I, Schuppe-Koistinen I, Ingelman-Sundberg $\mathrm{M}$ : The impact of CYP2E1 on the development of alcoholic liver disease as studied in a transgenic mouse model. J Hepatol 2008;50:572-583.

73 Bai J, Cederbaum AI: Adenovirus-mediated expression of CYP2E1 produces liver toxicity in mice. Toxicol Sci 2006;91:365-371. 